\title{
The kinetics of cluster fragmentation and depolymerisation
}

\author{
Robert M Ziff and E D McGrady \\ Department of Chemical Engineering, University of Michigan, Ann Arbor, MI 48109, USA
}

Received 31 January 1985

\begin{abstract}
The equation that describes fragmentation kinetics, such as that which occurs in cluster breakup and polymer chain degradation (depolymerisation), is solved for models where the rate of breakup depends upon the size of the object breaking up. The resulting dynamic scaling behaviour is investigated. Both discrete and continuous models are considered.
\end{abstract}

\section{Introduction}

The process of cluster formation has received considerable attention recently, ranging from the theoretical study of the kinetics of 'coagulation' (e.g. Hendriks et al 1983, Leyvraz 1984), to the computer simulations of 'cluster-cluster aggregation' (e.g. Meakin 1983, Kolb et al 1983). The many interesting things that have been studied include the explicit time-dependent distribution functions, the asymptotic (scaling) behaviour of those distributions, the appearance of singularities and gelation, and the fractal geometry of the clusters.

In this paper we consider the reverse process of cluster fragmentation, which occurs in cluster breakup, depolymerisation, rock fracture, droplet breakup, etc. We are interested in finding explicit expressions for the size distributions for models that are applicable to some of these processes.

In the field of polymer science, the fragmentation process has been of considerable interest, since degradation of bonds or depolymerisation results in fragmentation. In the early work of Kuhn (1930), Mark and Simha (1940), and Montroll and Simha (1940), the size distributions were found by statistical arguments. Jellinek and White (1951) and Saito (1958) introduced the kinetic equation of fragmentation, in the discrete and continuous forms, respectively, which allow the distributions to be found by analysis (although the equation for fragmentation in combination with coagulation was introduced earlier by Blatz and Tobobsky (1945)). Others who have considered the problem include Charlesby (1954) and Nanda and Pathria (1959). Almost all of this work has concerned the case of 'random scission'-all bonds break with equal probability, and the resulting size distribution, first found by Montroll and Simha (1940), has been rederived numerous times. In the work of Amemiya (1962) inhomogeneity was introduced by having bonds of different breaking probability dispersed throughout the system. Dependence of the scission rate on the size of the chain has been considered only recently in the work of Basedow et al (1978) and Ballauff and Wolf (1981), although explicit solutions were not found and the equations were solved by computer. 
Here, we are especially interested in models in which the breakup rate depends upon the size, in which case the cutting is non-random in the sense that bonds on different chains break with different probability, but random along any given chain. We consider mostly the continuous model, which is described by the kinetic equation (1) below. A continuous system continues to fragment without bound, and thus leads to long-time behaviour described by asymptotic, or scaling, exponents. The continuous case is also somewhat more interesting analytically, since the determination of the solutions (and indeed, their existence) is not quite as obvious as in the discrete case, where solutions can be found recursively. We consider the discrete equation only briefly, giving a simple derivation of the random-scission solution and a demonstration of what appears to be a new explicit solution for the case that the breakup rate is proportional to the length of the chain.

For a continuous system, the evolution of the distribution of sizes is described by the kinetic equation:

$$
\frac{\partial c(x, t)}{\partial t}=-c(x, t) \int_{0}^{x} F(y, x-y) \mathrm{d} y+2 \int_{x}^{x} c(y, t) F(x, y-x) \mathrm{d} y
$$

where $c(x, t)$ gives the concentration of $x$-mers as a function of time and $F(x, y)$ gives the intrinsic rate that an $(x+y)$-mer breaks up into an $x$-mer and a $y$-mer. The first term on the right-hand side represents the decrease in $x$-mers due to all possible breakups of an $x$-mer, $(x) \rightarrow(y)+(x-y), x>y$, and the second term represents the increase in $x$-mers due to reactions $(y) \rightarrow(x)+(y-x), y>x$, with a factor of 2 to take into account that either of the particles resulting can be an $x$-mer. (It is assumed that two particles are always produced.) For a given intrinsic fragmentation rate $F(x, y)$, and initial distribution $c(x, 0)$, (1) gives the complete time behaviour of $c(x, t)$. Equation (1) is the general form of the fragmentation equation written by Saito (1958), and is a limiting case of the coagulation-fragmentation equation (for zero coagulation rate) studied by Blatz and Tobolsky (1945), and more recently by Cohen and Benedek (1982) and van Dongen and Ernst (1984).

We consider models where $F(x, y)=(x+y)^{\alpha}$, in which the breakup depends upon the size of the object breaking up $(x+y)$, but not upon the individual sizes of the two pieces. In the language of chain polymers, this is the condition that the scission is independent of the position along the polymer, but is a function of the length of the chain (if $\alpha \neq 0$ ). Thus, a 10 -mer breaks up into a 9 -mer and a 1 -mer at the same rate as an 8 -mer and a 2 -mer. The case $\alpha=0$ corresponds to random scission. We give a special solution for all $\alpha$, and find complete solutions for a class of $\alpha$ which includes $\alpha=1,0,-\frac{1}{3},-\frac{1}{2},-\frac{3}{5}, \ldots,-1$. We first find the solution for $\alpha=0$ using a probabilistic argument, and for the other cases we use a method that allows the general solution to be found from the special one.

Note that (1) implies conservation of the total mass:

$$
\int_{0}^{\infty} x c(x, t) d x=\text { constant }
$$

More generally, it follows from (1) that the moments, defined by,

$$
M_{n}(t)=\int_{0}^{\infty} x^{n} c(x, t) \mathrm{d} x
$$


satisfy

$$
\frac{\mathrm{d} M_{n}}{\mathrm{~d} t}=\int_{0}^{\infty} \mathrm{d} x c(x, t) \int_{0}^{x} \mathrm{~d} y\left(2 y^{n}-x^{n}\right) F(y, x-y) .
$$

When $n=1$, the last term is zero by the symmetry of $F$, and this proves (2). For $F(x, y)=(x+y)^{\alpha}$, the moment equations become

$$
\mathrm{d} M_{n} / \mathrm{d} t=[(1-n) /(1+n)] M_{n+\alpha+1}
$$

which are trivially soluble for $\alpha=-1$, and for $M_{0}$ when $\alpha=0$.

\section{2. $F(x, y)=1$}

For this model, (1) becomes

$$
\frac{\partial c(x, t)}{\partial t}=-x c(x, t)+2 \int_{x}^{\infty} c(y, t) \mathrm{d} y .
$$

Here, the probability that an object breaks up into two pieces is independent of both the size of the object or of the pieces. For example, a system of (continuous) linear polymer chains whose bonds break randomly and independently is described by this model. By referring to this system of linear chains, we can find the solution to (6) by a simple probabilistic argument.

Let the initial state be one of uniform chains of length $l$ and imagine that the chains lie end-to-end on a single line. Since the cuts in the chain occur at random, they will be randomly placed, or Poisson distributed, along the entire length of this infinite line. The number of cuts is proportional to time, so the probability a given segment of length $\mathrm{d} x$ will be cut is given by $t \mathrm{~d} x$, and the number of cuts after a time $t$ will be on the average $t l$ (per original chain). From this information we can deduce the probability (per original chain) of finding a piece of a given length $x$.

A piece of length $x, x<l$, can come from either an end of the original chain, or from the middle. If it comes from the end, the probability $p(x) \mathrm{d} x$ that its length is between $x$ and $x+\mathrm{d} x$ is given by the probability there is a gap of length $x$, $\mathrm{e}^{-x t}$, multiplied by the probability that a cut occurs between $x$ and $x+\mathrm{d} x, t \mathrm{~d} x$ :

$$
p(x) \mathrm{d} x=2 t \mathrm{e}^{-x t} \mathrm{~d} x
$$

with a factor of two because the piece can come from either end of the original chain. For a piece in the middle two cuts are needed. The probability that the two cuts separated by a distance $x$ is given by

$$
t^{2} \mathrm{e}^{-x t} \mathrm{~d} x \mathrm{~d} y
$$

where $y$ is the position of one end. Integrating over $y$, which can range over an interval of length $l-x$, we find that the probability of finding a mid-piece of length $x$ is given by

$$
p(x) \mathrm{d} x=t^{2}(l-x) \mathrm{e}^{-x t} \mathrm{~d} x .
$$

Finally, the original chain remains whole with a probability that no cut occurs over its entire length, or $\mathrm{e}^{-1}$. Combining this with (7) and (9), we thus find that the mean number of segments of length $x$, or the size distribution, is given by

$$
c(x, t)=\mathrm{e}^{-i t} \delta(x-l)+\left[2 t+t^{2}(l-x)\right] \mathrm{e}^{-x t} \quad \text { for } x \leqslant l .
$$


The original chains disappear exponentially fast in time, and to take their place there appears an exponential distribution in $x$, multiplied by a linear term. As $t \rightarrow \infty, c \rightarrow l \delta(x)$. That (10) is a solution to (6) can be verified by substitution.

The general solution can now be written, because (6) is linear. For an initial distribution $c(l, 0)$, we find the general solution by multiplying $(10)$ by $c(l, 0)$ and integrating over all $l$, with the result

$$
c(x, t)=\mathrm{e}^{-t x}\left(c(x, 0)+\int_{x}^{\infty} c(y, 0)\left[2 t+t^{2}(y-x)\right] \mathrm{d} y\right) .
$$

This is a slightly simplified version of the expression given by Saito (1956). (In Saito's expression, the last term was written as a double integral.)

Note that Montroll and Simha's solution of the discrete version of this problem also involved a statistical derivation. They found, as in statistical mechanical arguments, the detailed probability of finding $n_{1}$ monomers, $n_{2}$ dimers, $n_{3}$ trimers, etc, and then averaged to find the mean distribution. In the method presented here, the probability of finding a piece of a given length, and thus its distribution, is found directly by assuming all cuts to be randomly distributed from the beginning.

\section{Alternate solution to $F=1$}

For an exponential initial distribution $c(x, 0)=\mathrm{e}^{-s x},(11)$ gives

$$
c(x, t)=(1+t / s)^{2} \mathrm{e}^{-x(s+t)},
$$

which remains an exponential distribution with an ever-increasing sharpness. One can verify directly that (12) is a solution to (6), or to the resulting partial differential equation,

$$
c_{t x}=-x c_{x}-3 c .
$$

Because of the linearity of (6), it follows that a linear combination, or an integral transform of (12),

$$
c(x, t)=\mathrm{e}^{-x t} \int_{0}^{\infty} f(s)[1+t / s]^{2} \mathrm{e}^{-s x} \mathrm{~d} s
$$

is also a solution to (6). Now we show that the general solution (11) can be found from the expression above. From (14) it follows that

$$
c(x, 0)=\int_{0}^{\infty} f(s) \mathrm{e}^{-s x} \mathrm{~d} s
$$

which implies

$$
\int_{x}^{\infty} c(y, 0) \mathrm{d} y=\int_{0}^{\infty} s^{-1} f(s) \mathrm{e}^{-s x} \mathrm{~d} s
$$

and

$$
\int_{x}^{\infty}(y-x) c(y, 0) \mathrm{d} y=\int_{0}^{\infty} s^{-2} f(s) \mathrm{e}^{-s x} \mathrm{~d} s .
$$


Expanding the bracketed term in (14) into $\left(1+2 t / s+t^{2} / s^{2}\right)$ and applying the identities (15)-(17), (11) follows immediately.

Of course, we had used (11) to find the exponential solution (12), and so found nothing new here. However, for other models this procedure will enable us to find new solutions.

\section{4. $F(x, y)=x+y$}

In this model, the rate of breakup is proportional to the size. In the linear polymer representation, it corresponds to the case where the probability that a bond breaks is proportional to the length of chain to which it is attached, and a simple statistical argument based upon independent cuts will not work. Equation (1) becomes

$$
\frac{\partial c(x, y)}{\partial t}=-x^{2} c(x, t)+2 \int_{x}^{x} y c(y, t) \mathrm{d} y
$$

or in PDE form

$$
c_{x t}=-x^{2} c_{x}-4 x c .
$$

Trying a solution in the form $c=A(t) \exp \left(-B(t) x^{n}\right)$ one can solve for $A, B$ and $n$ to find

$$
c(x, t)=(1+t / s) \exp \left[-(s+t) x^{2}\right]
$$

where $s=B(0)$. Write

$$
c(x, t)=\mathrm{e}^{-t x^{2}} \int_{0}^{\infty} f(s)(1+t / s) \mathrm{e}^{-s x^{2}} \mathrm{~d} s .
$$

By definition, when $t \rightarrow 0$,

$$
c(x, 0)=\int_{0}^{\infty} f(s) \mathrm{e}^{-s x^{2}} \mathrm{~d} s
$$

and it follows that

$$
\int_{x}^{\infty} 2 y c(y, 0) \mathrm{d} y=\int_{0}^{\infty} s^{-1} f(s) \mathrm{e}^{-s x^{2}} \mathrm{~d} s .
$$

Comparing (21) with (22) and (23), we find that the general solution is given by

$$
c(x, t)=\mathrm{e}^{-t x^{2}}\left(c(x, 0)+2 t \int_{x}^{\infty} y c(y, 0) \mathrm{d} y\right) .
$$

For example, for a monodisperse initial distribution, $c(x, 0)=\delta(x-l)$, we find

$$
c(x, t)= \begin{cases}2 t l \mathrm{e}^{-t x^{2}} & x<l \\ \delta(x-l) \mathrm{e}^{-t l^{2}} & x=l \\ 0 & x>l\end{cases}
$$

which can also be written

$$
c(x, t)=\mathrm{e}^{-t x^{2}}[\delta(x-l)+2 t l \theta(l-x)]
$$

where $\theta$ is the step function. As the delta function initial state disappears, a Gaussian 
forms. Compared to the previous model $(\alpha=0)$ where the distribution was exponential, this model shows a much slower production of small particles, as one would expect from the relative behaviour of the two breakup frequencies $F$.

For an exponential initial distribution, $c(x, 0)=\mathrm{e}^{-x},(24)$ yields

$$
c(x, t)=\exp \left(-t x^{2}-x\right)[1+2 t(1+x)] .
$$

5. $F(x, y)=(x+y)^{\alpha}$

Equation (1) becomes

$$
\frac{\partial c(x, t)}{\partial t}=-x^{\alpha+1} c(x, t)+2 \int_{x}^{\infty} y^{\alpha} c(y, t) \mathrm{d} y .
$$

Again, we have a special solution of the exponential form:

$$
c(x, t)=(1+t / s)^{2 /(\alpha+1)} \exp \left[-(s+t) x^{\alpha+1}\right] .
$$

It is convenient to introduce

$$
\begin{array}{lr}
m=2 /(\alpha+1) & \text { or } \alpha=(2-m) / m \\
z=x^{\alpha+1} & \chi(z, t)=c(x, t) .
\end{array}
$$

Then (29) becomes

$$
\chi(z, t)=(1+t / s)^{m} \exp [-(s+t) z]
$$

and the general solution can be found by writing

$$
\chi(z, t)=\mathrm{e}^{-t z} \int_{0}^{\infty} f(s)(1+t / s)^{m} \mathrm{e}^{-\mathrm{s} z} \mathrm{~d} s .
$$

We can rewrite (32) in terms of $\chi(z, 0)$ in closed form if we choose $m=1,2,3,4$, $5, \ldots$ These values of $m$ correspond to $\alpha=1,0,-\frac{1}{3},-\frac{1}{2},-\frac{3}{5}, \ldots$, the first two cases being just the solutions found above. Expanding the binomial $(1+t / s)^{m}$ and using the identities

$$
\begin{aligned}
& \chi(z, 0)=\int_{0}^{\infty} f(s) \mathrm{e}^{-s z} \mathrm{~d} z \\
& \int_{z}^{\infty}(y-z)^{n} \chi(y, 0) \mathrm{d} y=n ! \int_{0}^{\infty} s^{-n-1} f(s) \mathrm{e}^{-s z} \mathrm{~d} z
\end{aligned}
$$

we find that

or

$$
\chi(z, t)=\mathrm{e}^{-t z} \chi(z, 0)+t \mathrm{e}^{-t z} \int_{z}^{\infty} L_{m-1}^{(1)}[t(z-y)] \chi(y, 0) \mathrm{d} y
$$

$c(x, t)=\exp \left(-t x^{2 / m}\right)\left(c(x, 0)+\frac{2 t}{m} \int_{x}^{\infty} L_{m-1}^{(1)}\left[t\left(x^{2 / m}-y^{2 / m}\right)\right] y^{2 / m-1} c(y, 0) \mathrm{d} y\right)$

where we have introduced the associated Laguerre polynomial

$$
L_{m-1}^{(1)}(x)=\sum_{i=1}^{m} \frac{m !}{(m-i) ! i !} \frac{(-x)^{i-1}}{(i-1) !} .
$$


Equation (35) gives the general solution for all $m$. For example, for $m=3$ ( $F=$ $\left.(x+y)^{-1 / 3}\right)$, it gives

$c(x, t)=\mathrm{e}^{-x t^{2 / 3}}\left\{\delta(x-l)+(t / 3) l^{-1 / 3}\left[6+6 t\left(l^{2 / 3}-x^{2 / 3}\right)+t^{2}\left(l^{2 / 3}-x^{2 / 3}\right)^{2}\right]\right\}$.

The moments follow by integrating (35), and for $c(x, 0)=\delta(x-l)$ are given by

$$
M_{n}=l^{n} \boldsymbol{M}\left[(n-1)(m / 2),(n+1)(m / 2),-t l^{2 / m}\right]
$$

where $\boldsymbol{M}(a, b, z)$ is Kummer's confluent hypergeometric function (e.g. Abramowitz and Stegun 1964), which can be written in terms of elementary functions for certain $m$ and $n$. For $n=0$ and $m$ even, (38) is related to the Laguerre polynomials; for example, for $m=0, M_{0}=1+t l$, and for $m=2, M_{0}=1+t l^{1 / 2}+\frac{1}{6} t^{2} l$. For $m=3$, the moments are related to half-integer modified Bessel functions, ultimately expressible in exponentials, and so on.

The scaling behaviour is found by writing the asymptotic behaviour of $c(x, t)$ for small $x$ and large $t$ in the form

$$
c(x, t) \sim t^{w} x^{\top} \Phi\left(x t^{2}\right)
$$

where $\Phi(x) \rightarrow 1$ as $x \rightarrow 0$ and $\Phi(x) \rightarrow 0$ as $x \rightarrow \infty$, in analogy with the dynamic scaling behaviour that has been used for the coagulating systems for large $x$ and $t$ (Viscek and Family 1984). Mass conservation implies $w=z(\tau+2)$. The special solution (29) yields

$w=2 /(1+\alpha)=m, \quad \tau=0, \quad$ and $\quad z=1 /(1+\alpha)=\frac{1}{2} m$.

Solutions for initial conditions other than the special one will also give the same long-time behaviour, as long as they are well behaved as $x \rightarrow 0$. Otherwise, it is possible, by starting with a diverging (for small $x$ ) initial distribution, to influence the small- $x$ behaviour for all time, and thus change the exponents.

It follows from (39) that the moments evolve as $M_{n} \sim t^{z(1-n)}$, and in particular, $M_{0} \sim t^{z}$. As $m \rightarrow \infty$ in our class of models $(\alpha \rightarrow-1)$, the number of fragments thus grows faster and faster with time. Note that if we try to extend (40) for $\alpha<-1$, we find that $z$ becomes negative, implying a decrease in $M_{0}$, which makes no sense.

\section{6. $F(x, y)=(x+y)^{-1}$}

We can take the limit $m \rightarrow \infty$ in the model of $\S 5$ to find the solution for the case $\alpha=-1$, or $f(x, y)=1 /(x+y)$. Taking that limit in (36) (using that $x^{2 / m} \sim 1+(2 / m) \ln x$ ) we find

$$
c(x, t)=\mathrm{e}^{-t} c(x, 0)+(2 t)^{1 / 2} \mathrm{e}^{-t} \int_{x}^{x} \frac{I_{1}\left[2(2 t \ln (y / x))^{1 / 2}\right] c(y, 0) \mathrm{d} y}{y(\ln (y / x))^{1 / 2}} .
$$

For $c(x, 0)=\delta(x-l)$, the solution is given by

$$
\begin{aligned}
c(x, t) & =\mathrm{e}^{-t} \delta(x-l)+\frac{(2 t)^{1 / 2} \mathrm{e}^{-t} I_{1}\left[2(2 t \ln (l / x))^{1 / 2}\right]}{l(\ln (l / x))^{1 / 2}} \\
& =\mathrm{e}^{-t} \delta(x-l)+\frac{2 t \mathrm{e}^{-t}}{l} \sum_{i=0}^{\infty} \frac{[2 t \ln (l / x)]^{i}}{i !(i+1) !}
\end{aligned}
$$

(for $x \leqslant l$ ). We can multiply (41) by $x^{n}$ and integrate over all $x$ to find explicitly the 
$n$th moment

$$
M_{n}(t)=l^{n} \exp [(1-n) t /(1+n)]
$$

as given by the moment equation, (5). The zeroth moment, which represents the number of segments, is simply $M_{0}=\mathrm{e}^{t}$. The exponential growth follows because each chain, regardless of its length, breaks with equal probability (in contrast to the previous model, where each bond or element of the chain breaks with equal probability).

Indeed, the entire solution (42) can be understood from a probabilistic point of view. The first term in (42) represents the probability that an $l$-chain remains uncut. Rewrite the second term in (42) as

$$
\sum_{n=1}^{\infty} 2^{n}\left(\frac{t^{n} \mathrm{e}^{-t}}{n !}\right)\left(\frac{[\ln (l / x)]^{n-1}}{l(n-1) !}\right)
$$

the last factor above represents the probability that a piece is of length $x$ after exactly $n$ cuts, since it is equal to

$$
\int_{x}^{l} \frac{\mathrm{d} x_{n}}{x_{n}} \ldots \int_{x_{2}}^{l} \frac{\mathrm{d} x_{1}}{x_{1}} \int_{x_{1}}^{l} \frac{\mathrm{d} x}{x} \delta(x-l) .
$$

The middle factor in (44) gives the probability that after a time $t$, exactly $n$ cuts have been made, and the $2^{n}$ gives the degeneracy, or the number of pieces after $n$ generations of cuts.

For small time, (42) gives

$$
c(x, t)=\delta(x-l)+t[2 / l-\delta(x-l)]+t^{2}\left[\frac{1}{2} \delta(x-l)+(2 / l)(\ln (l / x)-1)\right] .
$$

To first order in time, the depleting chains form a uniform distribution (the $2 / l$ term). Already in second order, however, a logarithmic divergence at $x=0$ forms. As $x \rightarrow 0$ with $t$ fixed, (42) has the curious behaviour

$$
c(x, t) \sim l^{-1} \pi^{-1 / 2} t^{1 / 4} \mathrm{e}^{-t}(-2 \ln x)^{-3 / 4} \exp \left[2(-2 t \ln x)^{1 / 2}\right]
$$

which diverges, but is integrable.

\section{Discrete model}

In the case of a discrete system, the size distribution is represented by a function $c_{k}(t)$ which gives the time dependent concentration of $k$-mers $(k=1,2,3, \ldots)$, and satisfies the discrete kinetic equation,

$$
\frac{\mathrm{d} c_{k}}{\mathrm{~d} t}=-c_{k}(t) \sum_{i=1}^{k-1} F_{i, k-i}+2 \sum_{j=k+1}^{\infty} c_{j}(t) F_{k, j-k}
$$

where $F_{i j}$ gives the intrinsic rate that an $(i+j)$-mer breaks up into an $i$-mer and a $j$-mer. In principle, a solution can be found for any $F_{i j}$, since for a monodisperse initial state, (48) can be solved successively for $c_{l}, c_{l-1}, \ldots$, and the general solution then follows by linear combination. Of course, the solution may not be in a closed form, but for $F_{i j}=i+j$ we show below that a simple explicit solution exists. First we consider the case $F_{i j}=1$, and demonstrate that the Montroll-Simha solution can be found by a very simple probabilistic argument, analogous to the derivation for the continuous case given in $\$ 2$. 
For $F_{i, j}=1,(48)$ becomes

$$
\frac{\mathrm{d} c_{k}}{\mathrm{~d} t}=-(k-1) c_{k}(t)+2 \sum_{j=k+1}^{\infty} c_{j}(t) .
$$

This model corresponds to the case of linear polymers in which bonds break randomly, as in $\S 2$, but now for a discrete system.

To find the distribution, consider a monodisperse system of chains of length $l$, $c_{k}(t)=\delta_{k l}$, and suppose that after a certain period of time, a fraction $q$ of the bonds have been cut and $(1-q)$ have not. Thus $q$ is the extent of reaction. The probability of finding a chain of length $k, k \leqslant l$, will be given by

$$
c_{k}= \begin{cases}(1-q)^{l-1} & k=l \\ (1-q)^{k-1}\left[2 q+q^{2}(l-k-1)\right] & k<l\end{cases}
$$

for, analogous to (10), the first term gives the probability that an $l$-mer remains intact, the second gives the probability of finding a $k$-mer at either of the two ends of the original $l$-mer, and the last term gives the probability of finding a $k$-mer somewhere in the middle of the $l$-mer (there are $(l-k-1)$ such places).

The number of uncut bonds decreases exponentially in time: $1-q=\mathrm{e}^{-t}$. This result can be deduced from the behaviour of the zeroth moment, $M_{0}=\Sigma c_{k}$. For $c_{k}(0)=\delta_{k l}$, it follows from (49) that $M_{0}=l+(1-l) \mathrm{e}^{-t}$. By definition, $M_{0}=$ (the number of chains initially) + (the number of cuts), and since the former is 1 , it follows that the latter is $(l-1)\left(1-\mathrm{e}^{-t}\right)$. However, (the number of cuts $)=($ the number of possible cuts $=l-1)$ multiplied by (the probability of there being a cut $=q$ ). Thus, $q=1-\mathrm{e}^{-t}$.

That $(50)$ is a solution to (49) can be verified directly (although rather tediously). The solution for a general initial condition follows by taking a linear combination of solutions (50):

$$
c_{k}(t)=(1-q)^{k-1}\left(c_{k}(0)+\sum_{l=k+1}^{\infty} c_{l}(0)\left[2 q+q^{2}(l-k-1)\right) .\right.
$$

The discrete model also admits a solution analogous to (12). For an initial state $c_{k}(0)=a^{k-1}(1-a)^{2}$ (normalised so that $\Sigma k c_{k}=1$ ), where $a$ is a constant, (48) gives

$$
c_{k}(t)=(a p)^{k-1}(1-a p)^{2}
$$

where $p=\mathrm{e}^{-t}$. This initial state corresponds to the distribution found in a system of linear polymers formed by random condensation, where $a$ is the fraction of the bonds formed. The solution corresponds to the same distribution, with that fraction decreasing back to zero as $t \rightarrow \infty$. Such behaviour is expected since both the growth and the breakup are assumed to occur independently so their effect is the same: the bonds are random.

As in the continuous case, one can find the general solution (51) by writing $a=\mathrm{e}^{-s}$, multiplying (52) by $f(s)$, integrating over $s$, and relating the result to $c_{k}(0)$. However, this procedure does not seem to be generalisable to other discrete models, because for other models the necessary simple exponential solutions do not appear to exist.

For the case $F_{i j}=i+j$, a simple closed-form solution can be found by iteration, as follows. For $k=l,(48)$ gives $(\mathrm{d} / \mathrm{d} t) c_{l}=-l(l-1) c_{l}$, which implies

$$
c_{l}(t)=\exp [-l(l-1) t] \text {. }
$$


For $k=l-1,(48)$ gives $(\mathrm{d} / \mathrm{d} t) c_{l-1}=-(l-1)(l-2) c_{l-1}+2 l c_{l}$, or

$$
c_{l-1}(t)=[l /(l-1)]\{\exp [-(l-1)(l-2) t]+\exp [-l(l-1) t]\} \text {. }
$$

Continuing for $k=l-2, \ldots$, we find (due to very fortuitous cancellations) that the general formula is simply:

$$
c_{j}(t)=(l / j) \mathrm{e}^{-j^{2} t}\left(\mathrm{e}^{j t}-\mathrm{e}^{-j t}\right)
$$

for $1 \leqslant j<l$, which can easily be verified. The solution for an arbitrary initial condition follows by linear combination.

In the limit $l \rightarrow \infty, j \rightarrow \infty$, with $j / l \rightarrow x$ and $t l^{2} \rightarrow t^{\prime}$, the continuum result (26) for the model $F(x, y)=x+y$ is recovered. This is the limit of large chains and small time. For large time the behaviour of the discrete and continuous systems differ markedly: the continuous model fragments without limit, while the discrete system ends up as a stationary system of monomers.

\section{Conclusions}

By using a kinetic equation approach we have derived the evolution of a fragmenting system for some new models where the rate of fragmentation is a function of the size of the polymer, as well as for the simple model $(F=1)$ where the rate of breakup is independent of length. The latter model was solved by a very simple statistical argument. While such arguments work well when the events occur independently, for size dependent breakup frequencies the kinetic approach appears to be more straightforward. The problem is then reduced to solving an integro-differential equation.

These new models that we have solved have various applications. The case $F(x, y)=$ $x+y$ reflects a process where the rate of breakup increases with size, which might be expected to occur in polymers under tension, or in a destructive forcefield such as ultrasound. We have also solved this model in the discrete form. The model $F(x+y)=$ $1 /(x+y)$ corresponds to a case where every polymer has an equal probability of splitting, irrespective of length.

The equation presented here is rich in unexplored possibilities. It would be interesting to look at cases where the rate depends upon the size of the particles produced by the breakup, such as $F(x, y)=(x y)^{\alpha}$. Such a kernel would take into account a non-uniform distribution of products, as occurs in most droplet and colloidal systems upon breakup. For example, Basedow et al (1978) and Ballauff and Wolf (1981) have considered the case where $F(x, y)$ depends upon $(x-y)^{2}$, and the resulting distribution (found numerically) was found to describe the experimental observations more closely than the independent model, $F=1$.

We noted from the scaling relations that as $\alpha \rightarrow-1, M_{0}$ grows faster with time, and ultimately, when $\alpha=-1, M_{0}$ grows exponentially. Can this be the forerunner of singular solutions? In the case of coagulation kinetics, when the coagulation rate is fast enough (the kernel has a homogeneity greater than 1), gelation occurs, marked by the formation of an infinite cluster in a finite amount of time. Models at the boundary of gelation (coagulation kernel $K(x, y)=x+y$, for example) show exponential growth in the mean size, analogous to the $\alpha=-1$ model here, where the number of particles grow exponentially. Perhaps a model where $F(x, y)$ grows more rapidly than $(x+y)^{-1}$ as $x, y \rightarrow 0$ will show an analogous phenomenon, in which a finite fraction of the mass of the system will belong to particles of zero size. 
Note added. Some of the above questions will be considered in a forthcoming publication (Ziff and McGrady 1985).

\section{Acknowledgments}

This paper is dedicated to the memory of Mark Kac. RMZ gratefully acknowledges support from the Shell Foundation as a Shell Faculty Fellow, and EDM acknowledges support from the Rockwell Foundation as a Rockwell Fellow. The authors thank Dr $M$ Terrill for pointing out the reference to the work of Basedow, Ebert and Ederer.

\section{References}

Abramowitz M and Stegun I 1964 Handbook of Mathematical Functions (Washington, DC: National Bureau of Standards)

Amemiya A 1962 J. Phys. Soc. Japan 17 1245, 1694

Ballauff M and Wolf B A 1981 Macromolecules 14654

Basedow A M, Ebert K H and Ederer H J 1978 Macromolecules 11774

Blatz P J and Tobolsky A V 1945 J. Phys. Chem. 4977

Charlesby A 1954 Proc. R. Soc. A 224120

Cohen R J and Benedek G B 1982 J. Phys. Chem. 863696

Hendriks E M, Ernst M H and Ziff R M 1983 J. Stat. Phys. 31519

Jellinek H H G and White G $1951 \mathrm{~J}$. Polym. Sci. 6745

Kolb M, Botet R and Jullien R 1983 Phys. Rev. Lett. 511123

Kuhn W 1930 Ber. Chem. Disch. Ges. 631502

Leyvraz F 1984 Phys. Rev. A 29854

Mark H and Simha R 1940 Trans. Faraday Soc. 35611

Meakin P 1983 Phys. Rev. Lett. 511119

Montroll E W and Simha R 1940 J. Chem. Phys. 8721

Nanda V S and Pathria R K 1959 J. Chem. Phys. 3027

Saito O 1958 J. Phys. Soc. Japan 13198

van Dongen P and Ernst M H 1984 J. Stat. Phys. 37301

Viscek T and Family F 1984 Phys. Rev. Lett. 521669

Ziff R M and McGrady E D 1985 to be published 